\title{
A CRITERION FOR THE POSITIVITY OF SINE POLYNOMIALS
}

\author{
JOHN STEINIG
}

\begin{abstract}
A sufficient condition is derived for a sine poly-
\end{abstract} nomial with real coefficients to be nonnegative on $(0, \pi)$.

We consider sine polynomials with real coefficients, and prove the following theorem.

\section{THEOREM. If}

$$
F(x)=A_{1} \sin x+A_{2} \sin 2 x+\cdots+A_{n} \sin n x,
$$

and if

(2) $A_{k} \geqq 2 \sum_{v=1}^{n-k}(-1)^{v+1} A_{k+v}, \quad k=1, \cdots, n-1$, and $A_{n}>0$, then

$$
F(x) \geqq 0 \text { for } 0<x<\pi .
$$

Inequality (3) is strict on $(0, \pi)$ if and only if $\left(k_{1}, \cdots, k_{s}, n\right) \leqq 2$, where $k_{1}, \cdots, k_{s}$ are those $k, 1 \leqq k \leqq n-1$, for which strict inequality holds in (2), and $\left(k_{1}, \cdots, k_{s}, n\right)$ is their greatest common divisor with $n$.

Proof. For any $n \geqq 1$, we have

$$
\sigma_{n}(x):=\sum_{v=1}^{n} \sin \left(v-\frac{1}{2}\right) x \geqq 0, \quad 0<x<2 \pi,
$$

and therefore also

$$
S_{n}(x):=\sum_{v=1}^{n} b_{v} \sin \left(v-\frac{1}{2}\right) x \geqq 0, \quad 0<x<2 \pi,
$$

if

$$
b_{1} \geqq b_{2} \geqq \cdots \geqq b_{n}>0,
$$

as a summation by parts will show:

$$
S_{n}(x)=\sum_{k=1}^{n-1}\left(b_{k}-b_{k+1}\right) \sigma_{k}(x)+b_{n} \sigma_{n}(x) .
$$

Received by the editors March 24, 1972 and, in revised form, September 21, 1972. AMS (MOS) subject classifications (1970). Primary 42A04, 33A10, 33A65, 26 A82.

Key words and phrases. Trigonometric polynomials, Jacobi polynomials, positivity.

(c) American Mathematical Society 1973 
Hence, if (6) is satisfied, we have

that is,

$$
\sum_{v=1}^{n} b_{v} \sin \left(v-\frac{1}{2}\right) x \cos \frac{x}{2} \geqq 0, \quad 0<x<\pi,
$$

or again,

$$
\sum_{v=1}^{n} b_{v}\{\sin (v-1) x+\sin v x\} \geqq 0,
$$

where

$$
\sum_{v=1}^{n} A_{v} \sin v x \geqq 0, \quad 0<x<\pi,
$$

$$
A_{v}=b_{v}+b_{v+1} \text { for } 1 \leqq v \leqq n-1 \text {, and } A_{n}=b_{n} .
$$

Now if the $A$ 's and the $b$ 's are related as in (8), then conditions (2) and (6) are equivalent. Indeed, (2) can be written as

$$
b_{k}+b_{k+1} \geqq 2 \sum_{v=1}^{n-k-1}(-1)^{v+1}\left(b_{k+v}+b_{k+v+1}\right)+2(-1)^{n-k+1} b_{n}=2 b_{k+1}
$$

if $k \leqq n-1$, and if $k=n, b_{n}=A_{n}>0$. This completes the proof of (3).

The determination of cases of equality in (3) rests on their determination in (5); for this we require the familiar identity

$$
\sigma_{n}(x)=\sin ^{2}(n x / 2) / \sin (x / 2) .
$$

Let $k_{1}, \cdots, k_{s}$ be those indices $k$ for which $b_{k}>b_{k+1}$. It is obvious from (7) and (9) that the zeros of $S_{n}(x)$ are the common zeros of

$$
\sin \left(k_{1} x / 2\right), \cdots, \sin \left(k_{s} x / 2\right), \sin (n x / 2) .
$$

And the common zeros of these functions are at $x=2 m \pi /\left(k_{1}, \cdots, k_{s}, n\right)$, $m \in Z$, where $\left(k_{1}, \cdots, k_{s}, n\right)$ denotes the greatest common divisor of $k_{1}, \cdots, k_{s}, n$. Thus, these functions have a common zero on $(0, \pi)$ if and only if $\left(k_{1}, \cdots, k_{s}, n\right)>2$. This observation concerning (5), together with the relation between $A$ 's and $b$ 's, yields the statement concerning equality in (3). The proof of the Theorem is now complete.

Corollary. Let $F$ be defined as in (1). If $A_{1} \geqq A_{2} \geqq \cdots \geqq A_{n-1} \geqq$ $2 A_{n}>0$, if $2 A_{k} \leqq A_{k-1}+A_{k+1}$ for $k=2, \cdots, n-2$, and if $2 A_{n-1} \leqq A_{n-2}+$ $2 A_{n}$, then $F(x) \geqq 0$ for $0<x<\pi$.

This is a theorem of Fejér [4, Satz 27]; it is easily obtained as a corollary of the theorem we have just proved by verifying that Fejér's conditions (that the sequence $A_{1}, \cdots, A_{n-1}, 2 A_{n}$ be positive, nonincreasing and convex) imply conditions (2). 
Examples show that Fejér's conditions are more restrictive than those in (2). For instance, the Theorem implies the positivity of $4 \sin x+$ $3 \sin 2 x+(2-\varepsilon) \sin 3 x+\frac{1}{2} \sin 4 x$ for $0<x<\pi$ and $0 \leqq \varepsilon \leqq \frac{1}{2}$, but the Corollary applies only to the case $\varepsilon=0$.

The argument used to prove the Theorem shows that

if and only if

$$
\sum_{v=1}^{n} a_{v} \sin (2 v-1) x \geqq 0, \quad 0<x<\pi,
$$

$$
\sum_{v=1}^{n} \alpha_{v} \sin v x \geqq 0, \quad 0<x<\pi,
$$

where $\alpha_{v}=a_{v}+a_{v+1}$ for $v=1, \cdots, n-1$, and $\alpha_{n}=a_{n}$.

On the other hand, a similar argument shows that (10) holds if and only if

$$
\frac{1}{2} \lambda_{0}+\sum_{v=1}^{n-1} \lambda_{v} \cos v x \geqq 0, \quad 0<x<2 \pi,
$$

where $a_{v}=\lambda_{v-1}-\lambda_{v}, v=1, \cdots, n-1$, and $a_{n}=\lambda_{n-1}$ (multiply the cosine polynomial in (11) by $\sin x / 2$ ). This shows that the Theorem we have proved is equivalent to a theorem of Fejér [3, Theorem II] which states that (11) holds if the sequence $\lambda_{0}, \lambda_{1}, \cdots, \lambda_{n}, 0,0$ is convex and the $\lambda$ 's are positive. In fact, one of Fejér's proofs of his theorem [5] is quite similar to the proof we have given of (3).

Turán has found another consequence of (10), namely

$$
\sum_{v=1}^{n} \frac{a_{v}}{v} \geqq 0, \quad 0<x<\pi,
$$

with strict inequality unless all $a$ 's vanish ([1], [6], [7]).

These results can be placed in a more general setting by considering the Jacobi polynomials $P_{n}^{(\alpha, \beta)}$, defined by

$$
(1-x)^{\alpha}(1+x)^{\beta} P_{n}^{(\alpha, \beta)}(x)=\frac{(-1)^{n}}{2^{n} n !} \frac{d^{n}}{d x^{n}}\left[(1-x)^{n+\alpha}(1+x)^{n+\beta}\right],
$$

$\alpha, \beta>-1[8]$. Since

$$
P_{n}^{(1 / 2,-1 / 2)}(\cos \theta) / P_{n}^{(-1 / 2,1 / 2)}(1)=\sin \left(n+\frac{1}{2}\right) \theta / \sin \frac{1}{2} \theta,
$$

(4) is the same as

$$
\sum_{k=0}^{n} \frac{P_{k}^{(\alpha, \beta)}(x)}{P_{k}^{(\beta, \alpha)}(1)} \geqq 0, \quad-1<x<1,
$$

for $\alpha=\frac{1}{2}, \beta=-\frac{1}{2}$. R. Askey [2] has shown that (12) holds for all $n \geqq 0$ if 
$\alpha+\beta \geqq 0,-1<\alpha \leqq \beta+1$. Now if (12) holds, so does

$$
\sum_{k=0}^{n} b_{k} \frac{P_{k}^{(\alpha, \beta)}(x)}{P_{k}^{(\beta, \alpha)}(1)} \geqq 0, \quad-1<x<1,
$$

if $b_{0} \geqq b_{1} \geqq \cdots \geqq b_{n}>0$ (sum by parts); for $\alpha=\frac{1}{2}, \beta=-\frac{1}{2}$, this is (5).

In this setting, our proof of (3) can be formulated thus: (3) follows from (13) and the recurrence relation

$$
\frac{P_{n}^{(\alpha, \beta)}(x)}{P_{n}^{(\beta, \alpha)}(1)}+\frac{P_{n+1}^{(\alpha, \beta)}(x)}{P_{n+1}^{(\beta, \alpha)}(1)}=\frac{(2 n+\alpha+\beta+2)}{(2 \beta+1)}(1+x) \frac{P_{n}^{(\alpha, \beta+1)}(x)}{P_{n}^{(\beta+1, \alpha)}(1)}
$$

on setting $\alpha=\frac{1}{2}, \beta=-\frac{1}{2}$, since

$$
P_{n}^{(1 / 2,1 / 2)}(\cos \theta) / P_{n}^{(1 / 2,1 / 2)}(1)=\sin (n+1) \theta /(n+1) \sin \theta .
$$

\section{REFERENCES}

1. R. Askey, J. Fitch and G. Gasper, On a positive trigonometric sum, Proc. Amer. Math. Soc. 19 (1968), 1507. MR 37 \#6662.

2. R. Askey, Positive Jacobi polynomial sums, Tôhoku Math. J. 24 (1972), 109-119.

3. L. Fejér, Über die positivität von Summen, die nach trigonometrischen oder Legendreschen Funktionen fortschreiten (erste Mitteilung), Acta Litt. ac Sci. Szeged 2 (1925), 75-86 (reproduced in Gesammelte Arbeiten II, Birkhäuser Verlag, Basel, 1970, pp. 128-138).

4. - Einige Sätze, die sich auf das Vorzeichen u.s.w., Monatsh. Math. Phys. 35 (1928), 305-344 (reproduced in Gesammelte Arbeiten II, 202-237).

5. - U Über ein trigonometrisches Analogon eines Kakeyaschen Satzes, Jber. Deutsch. Math.-Verein. 38 (1929), 231-238 (reproduced in Gesammelte Arbeiten II, 256-263).

6. C. Hyltén-Cavallius, A positice trigonometrical kernel, Tolfte Skand. Mat. Kongr., 1953, Lund (1954), 90-94. MR 16, 353.

7. P. Turán, On a trigonometrical sum, Ann. Soc. Polon. Math. 25 (1952), 155-161. MR 14, 1080.

8. G. Szegö, Orthogonal polynomials, Amer. Math. Soc. Colloq. Publ., vol. 23, rev. ed., Amer. Math. Soc., Providence, R.I., 1959. MR 21 \#5029.

Section de Mathématiques, Université de Genève, 1211 Genève 24, Switzerland 